\title{
Le parfum de l'homme éthique, et autres questions touchant à la morale dans l'enseignement, la langue et les textes
}

\section{Marie-Anne Paveau}

\section{OpenEdition}

\section{Journals}

Édition électronique

URL : http://journals.openedition.org/pratiques/2236

DOI : $10.4000 /$ pratiques.2236

ISSN : 2425-2042

Éditeur

Centre de recherche sur les médiations (CREM)

\section{Édition imprimée}

Date de publication : 31 décembre 2014

\section{Référence électronique}

Marie-Anne Paveau, "Le parfum de l'homme éthique, et autres questions touchant à la morale dans l'enseignement, la langue et les textes », Pratiques [En ligne], 163-164 | 2014, mis en ligne le 31 décembre 2014, consulté le 19 avril 2019. URL : http://journals.openedition.org/pratiques/2236 ; DOI : 10.4000/pratiques.2236

Ce document a été généré automatiquement le 19 avril 2019.

(c) Tous droits réservés 


\title{
Le parfum de l'homme éthique, et autres questions touchant à la morale dans l'enseignement, la langue et les textes
}

\author{
Marie-Anne Paveau
}

« De petite valeur est le parfum du tagara ou du santal. Le parfum de l'homme éthique, qui se répand même parmi les Devas, est excellent » (Les dits du Bouddha) « Mais il faut comprendre que la moralité ne vient pas comme une couche secondaire, au-dessus d'une réflexion abstraite sur la totalité et ses dangers ; la moralité a une portée indépendante et préliminaire. La philosophie première est une éthique » (Emmanuel Lévinas, Éthique et infini).

\section{La morale : où est le problème?}

1 Il est des mots dont le sort est scellé avant même d'avoir été contextualisés ; c'est le cas de morale, qui dégage aussitôt, en France en tout cas, et dans les milieux éducatifs, un puissant halo connotatif, où se croisent de manière stéréotypée l'ordre religieux et la contrainte sociale, la réprimande et la culpabilisation. Dans ce halo sémantique, naviguent des compositions et des figements, qui ne vont pas non plus vers l'ouverture et la tolérance : ordre moral, cours de morale, morale religieuse, autant de segments situés dans des lignées discursives un peu sombres, et parfois de triste mémoire.

Mais ce mot tant haï et si vite associé aux préaux et aux blouses grises, recouvre en fait un continent riche et varié de conceptions et de pensées qui engagent les fondements de la vie humaine : le bien et le mal, le juste et l'injuste, les valeurs et les normes, les contrats sociaux, la reconnaissance, le rapport à l'autre, la vie commune, le commun, le partage, le 
respect, l'honnêteté. Pour ces raisons, et parce que je crois en la réappropriation, versant linguistique de la resignification que nous a enseignée J. Butler (2004 [1997]), j’ai choisi d'intituler Langage et morale mon travail sur l'intégration de la dimension normative et subjective à la théorie du discours (Paveau, 2013). Ma position s'inspirait de la moral philosophy anglophone, qui ne connait pas ces réserves idéologiques, et qui aborde la question des faits et des comportements, des valeurs et des normes, avec une liberté que la pensée française n'a pas, ou plus.

Quand A. Petitjean m'a proposé d'élaborer un numéro de Pratiques sur la mise au travail de la notion de morale dans les domaines de prédilection et de spécialité de cette revue désormais quadragénaire, je me suis dit qu'il était peut-être temps, dans nos discours académiques si prompts à se fermer aux risques d'hétérodoxie ou d'idéologie, de questionner les normes et les valeurs morales. Après tout, 40 ans, n'est-ce pas l'âge de la maturité et du début de la sagesse? Pour travailler la question morale, la revue Pratiques est en outre un lieu idéal puisqu'elle concerne les domaines sémiotiques privilégiés de la dimension morale, tant en ce qui concerne les productions (textes, discours, cours, manuels, formes langagières, etc.) que les travaux réflexifs (analyse du texte et du discours, didactique, analyse des textes littéraires, normes de littérarité, etc.).

\section{Morale laïque, une expression polémique}

C'est non seulement un lieu idéal, mais un lieu où les enseignant-e-s, de français tout particulièrement mais aussi d'autres disciplines, pourront trouver des pistes de réponses à l'épineuse " affaire de la morale laïque ", et, de manière plus générale, à la question de l'enseignement des valeurs et des normes en contexte public (au sens institutionnel du terme). Je rappelle brièvement le contenu de la polémique qui a éclaté en France en septembre 2012, au moment où le ministre de l'Éducation nationale de l'époque, Vincent Peillon, exposait ses conceptions de la morale et de son enseignement dans une interview au journal du dimanche :

«JDD. Qu'entendez-vous par "morale laïque" ?

Vincent Peillon. La morale laïque, c'est comprendre ce qui est juste, distinguer le bien du mal, c'est aussi des devoirs autant que des droits, des vertus, et surtout des valeurs. Je souhaite pour l'école française un enseignement qui inculquerait aux élèves des notions de morale universelle, fondée sur les idées d'humanité et de raison. La république porte une exigence de raison et de justice. La capacité de raisonner, de critiquer, de douter, tout cela doit s'apprendre à l'école. Le redressement de la France doit être un redressement matériel mais aussi intellectuel et moral $»^{1}$.

5 Son prédécesseur Luc Chatel réagit immédiatement à l'expression de «redressement intellectuel et moral » en comparant, dans un tweet devenu célèbre, Vincent Peillon à Pétain qui, dans un discours de juin 1940, déclarait : «C'est à un redressement intellectuel et moral que, d'abord, je vous convie. » Au-delà de la polémique et du recours à une forme de point Godwin, désormais entré dans les mœurs discursives politiques et médiatiques, c'est tout le réseau de connotations négatives du terme moral qui est convoqué, et la question même de la possibilité de la morale à l'école qui est suscitée. Les débats qui s'ensuivent permettent cependant d'approfondir la question et de tracer une généalogie des conceptions de la morale à l'école, et, plus largement, de raccrocher cette polémique aux grandes questions de la philosophie morale. 
6 C. Lelièvre par exemple, dans un billet du blog Histoire et politique scolaire qu'il tient sur la plateforme de Mediapart, montre que la conception de la morale selon Vincent Peillon est proche de celle de Ferdinand Buisson :

«En réalité le ministre de l'Education nationale est bien plus proche de la position de Ferdinand Buisson, qu'il connaît très bien puisqu'il lui a consacré un livre entier (qu'il considère même comme son livre majeur) : "Une religion pour la République" en écho au livre de Ferdinand Buisson, "La foi laïque" " (Lelièvre, 2012).

7 C. Lelièvre insiste sur un point précis de la conception de Ferdinand Buisson, l'intuition morale : «Et Ferdinand Buisson comptait explicitement sur les possibilités de "l'intuition morale", les interrogations et les possibilités de la "conscience" individuelle » (Lelièvre, 2012). C'est un point important des débats sur la question morale en philosophie : la morale est-elle naturelle? Sommes-nous nés ou devenus moraux? Je vais revenir sur ce point plus bas, mais je rappelle déjà que l'idée d'une conscience morale de l'individu n'est pas nouvelle, qu'elle se trouve formulée chez Aristote qui expose une morale de la vertu et des dispositions dans L'éthique à Nicomaque, et qu'elle court dans toute la conception du sens moral du XVIII ${ }^{\mathrm{e}}$ siècle (Locke, Shaftesbury, Hume, Kant). Patrick Cabanel, historien interviewé dans Le Monde, a également un avis dépassionné dans cette polémique de la rentrée 2012, trouvant que l'apprentissage du vivre-ensemble a bien des fondements moraux et que ce n'est pas scandaleux de le souligner :

"Retour à la morale, ça sonne réactionnaire, de droite. Pourtant, je ne suis pas sûr qu'il y ait une différence entre "morale" et "morale laïque". Les ministres de l'éducation successifs renvoient à la même chose : l'impératif catégorique kantien, qui pourrait se résumer par "Ma liberté commence là où s'arrête celle d'autrui". "

"Lorsqu'en 1882, l'instruction laïque et morale a remplacé l'instruction religieuse et morale, "Tu aimeras ton prochain comme toi-même" est devenu "Ne fais pas à autrui ce que tu ne veux pas qu'il te fasse". C'est un autre habillage, mais c'est la même question fondamentale. C'est le noyau universel, intouchable. Ce n'est pas une morale de gendarme $»^{2}$.

8 L'idée d'un « noyau universel » est intéressante, et fera d'ailleurs l'objet de la critique de R. Ogien détaillée plus bas. Si l'on observe de près les paroles de Vincent Peillon, on relève des mots et expressions qui font partie du lexique le plus banal de la philosophie morale : morale universelle, humanité, raison, connaissance, dévouement, solidarité, égalité, juste, bien, mal, devoirs, droits, vertus, valeurs. Son ambition est de proposer des enseignements à propos de «toutes les questions que l'on se pose sur le sens de l'existence humaine, sur le rapport à soi, aux autres, à ce qui fait une vie heureuse ou une vie bonne " (extrait de la même interview pour Le JDD). La vie bonne, c'est le concept moral fondamental, d'Aristote à Ricœur en passant par Hume, Kant, Rawls, Williams, Levinas. Regardé à partir du corpus imposant de la philosophie morale depuis l'Antiquité, le discours de Vincent Peillon parait alors ordinaire, et même assez plat et peu inventif sur le plan des idées; vu à travers un prisme plus politique et plus idéologique, il semble s'inscrire dans un courant conservateur, voire réactionnaire, celui d'un « redressement » de ce qui est donc censé être tombé, et s'intégrer dans les formes bien connues, aussi qu'increvables du discours déploratoire et décliniste que M. Angenot a si bien décrit dans La parole pamphlétaire (Angenot, 1982).

9 Mais qu'en est-il du contenu du rapport commandé par le ministre en octobre 2012, et rendu en avril 2013, Morale laïque. Pour un enseignement laïque de la morale 3 ? Le contenu est désormais bien connu et je ne vais pas le résumer, mais plutôt noter quelques points qui intéressent directement ce numéro. D'abord, les auteurs du rapport insistent à plusieurs 
reprises sur la double dénomination morale laïque et civique, situant donc son objet dans le champ de la vie de la cité (ce qui implique une dimension en même temps sociale et politique), du vivre-ensemble, et de qui est appelé un faire communauté: "La morale enseignée à l'École ne peut être qu'une morale laïque en ce qu'elle est non confessionnelle et une morale civique en ce qu'elle est en lien étroit avec les principes et les valeurs de la citoyenneté républicaine et démocratique » (p. 23). Ensuite, le rapport opère un choix clair entre les deux grandes options dont tout un chacun dispose en matière de morale, les normes ou les valeurs. Les auteurs du rapport sont du côté d'une éthique ${ }^{4}$ des valeurs, c'est-à-dire d'une conception plutôt interne et intersubjective du vivre-ensemble, dont les règles sont négociées de manière immanente, et non de celui d'une éthique des normes, à la Kant, pour laquelle nos comportements seraient prescrits par des règles externes et transcendantes (l'impératif catégorique). La notion de valeur est définie dans le rapport de manière très intéressante sous l'angle de sa transmission :

"Une deuxième condition touche la nature même de ce qu'on appelle "valeur". Les enseignants sont souvent embarrassés dans la transmission des valeurs, soit qu'ils engagent les élèves dans des exercices intellectuels dont les retombées pratiques restent aléatoires, soit qu'ils transmettent ces valeurs dans l'ordinaire de la classe, sans être toujours certains que les élèves en aient bien compris et mesuré l'importance. Dans ce cadre, il faut repréciser que ce qu'on appelle "valeur" comprend trois dimensions : une dimension intellectuelle (la valeur a un contenu cognitif), une dimension psycho-affective (elle a du prix, elle vaut quelque chose pour celui qui s'en réclame), une dimension conative (elle oriente et irrigue les conduites et les engagements). C'est dans ces trois directions qu'il faut orienter la transmission des valeurs à l'École: la première engage des contenus intellectuels qui trouveront à s'alimenter, par exemple, de l'étude de textes. Un travail sur la peine de mort mobilisant les valeurs de la dignité et de la justice pourra en ce sens être mené à partir de textes fondateurs du questionnement éthique et juridique sur la peine de mort, ceux de Beccaria, Voltaire, Victor Hugo, Lamartine ou Robert Badinter.

Un travail sur la dimension psycho-affective des valeurs a un double objectif: permettre aux élèves de mettre à distance leurs propres valeurs, croyances et préjugés (par exemple, des croyances racistes ou des préjugés sexistes), rendre désirables les valeurs communes. Des supports plus sensibles comme les supports artistiques, les films notamment, sont ici préférables même s'ils n'interdisent pas un travail intellectuel.

La dimension conative des valeurs concerne l'action. Les situations d'autonomie, de coopération, de responsabilité, de participation doivent viser à faire vivre les valeurs communes mais aussi à les rendre désirables. En ce sens, il convient non seulement de favoriser, mais surtout de valoriser l'engagement des élèves dans la communauté » (p. 29).

10 Le terme désirable plusieurs fois utilisé dans ce passage est celui de la définition la plus classique des valeurs dans le corpus philosophique, place la morale du côté des individus et de leur communauté, et met à distance les notions de contrainte et d'imposition souvent attribuées, à raison d'ailleurs, à l'éthique des normes fondant des morales prescriptives. Une petite phrase du rapport pose exactement cette question et ouvre sur la définition même de l'éducation: "La marge est étroite ici entre imposer et faire respecter: c'est l'espace de l'éducation»(p. 28). Imposer, du côté de l'exercice du pouvoir, et faire respecter, du côté de l'apprentissage des valeurs. Enfin, sur la question très sensible d'une éventuelle " pédagogie de la morale », le rapport propose des objectifs qui, rédigés de manière un peu lyrique, n'en sont pas moins éclairants sur la perspective d'une morale laïque qui ne serait pas prescriptive : «Le rôle de l'enseignant n'est donc 
pas de proposer "une morale" mais de conduire les élèves à développer le courage de penser, la passion de comprendre, la volonté de s'engager » (p. 33).

\section{La critique de R. Ogien}

11 Ce rapport et les conceptions et objectifs qui le sous-tendent ont été largement critiqués, l'opposition la plus intéressante et la plus synthétique étant celle que R. Ogien a exposée dans un ouvrage, La guerre aux pauvres commence à l'école. Sur la morale laïque (Ogien, 2013). Sa position est que le projet de la morale à l'école est une idée socialement conservatrice, philosophiquement confuse et politiquement dangereuse.

Socialement conservatrice. Pour R. Ogien, le problème principal de l'école est matériel et économique. L'école est une chambre d'écho des inégalités socioéconomiques, qu'elle contribue à renforcer, au lieu de les compenser. La solution ne passe donc pas par l'éducation des dispositions morales des élèves, dont le déficit n'a selon lui pas grandchose à voir avec le coefficient de justice à l'œuvre dans notre société. Le projet de Vincent Peillon lui semble sous-tendu par l'idée que les difficultés sociales découleraient de l'immoralité des élèves les plus défavorisés : «En fait, ce qu'on appelle "incivilités", c'est, la plupart du temps, certaines conduites agressives quand elles sont le fait des plus pauvres, des classes dites “dangereuses" » (Ogien, $2013: 48$ ).

Philosophiquement confuse. R. Ogien considère que l'apprentissage de la pensée rationnelle et de la pensée libre ne garantissent en aucun cas le respect des valeurs morales positives :

« Le projet d'instaurer des cours de morale laïque à l'école, comme l'actuel ministre de l'Education nationale le conçoit, est une illustration presque parfaite de la forme la plus courante de naïveté épistémologique, celle qui consiste à croire que la libre discussion ou la réflexion rationnelle aboutiront nécessairement à un accord sur les valeurs » (Ogien, $2013: 110)$.

De plus, la question de l'enseignabilité de la morale se pose de manière aigüe selon lui. Comme il le souligne dans les nombreuses interviews qu'il a accordées dans la presse à propos de ce livre, l'enseignement de la morale ressemble plus à celui de la natation qu'à celui des mathématiques, ce qui pose de redoutables problèmes d'évaluation; il semble peu pertinent, en effet, d'appliquer à la morale laïque les règles d'évaluation, qu'elles soient formatives ou sommatives, des disciplines traditionnellement enseignées à l'école.

Politiquement dangereuse. Pour R. Ogien, parler de la morale laïque au singulier est une grossière erreur car c'est faire peu de cas de l'hétérogénéité des conceptions de la laïcité, des morales qui peuvent être rangées sous cette étiquette, et des relativités culturelles qui peuvent s'y abriter. Le mot laïcité lui semble un piège politique permettant de promouvoir une culture de la francité (représentés par «le vin et le cochon », selon son expression) et de stigmatiser et exclure des minorités religieuses ayant des pratiques vestimentaires et culinaires «non conformes». C'est donc ce singulier qui lui semble représenter un véritable danger politique, car il recèle selon lui une inaptitude à la diversité et à la tolérance. 


\section{Brève incursion dans le domaine étendu de la philosophie morale}

$$
\text { plus en détail, mais en attendant je propose un très bref parcours dans le corpus de la }
$$
philosophie morale telle qu'elle se présente à nous actuellement.

Tout d'abord un point sur ce qui est presque une scie terminologique dans le domaine: doit-on parler de morale ou d'éthique ? Ces deux mots impliquent-ils des différences ou une équivalence? Il est vrai que le mot éthique, pourtant omniprésent dans de nombreux secteurs de la société, ne déclenche pas les débats et polémiques vus précédemment. Plus neutre et d'apparence plus technique et scientifique, il permet souvent d'éviter morale, imprégné de religiosité et de prescriptivité, comme on l'a vu. La différence sémantique entre les termes est aussi, parfois, un dispositif argumentatif : ce qui est accepté sous le nom éthique est refusé sous celui de morale. . La distinction, sur le plan philosophique, se trouve dans un ensemble de textes assez contradictoires qui développent des distinctions peu homogènes, à tel point que les mêmes raisons peuvent plaider pour l'un ou l'autre emploi (sur ce point, voir Paveau, 2013). L'éthique serait en effet du côté de la théorie et de la réflexion, dans une perspective universaliste, et la morale du côté des applications pratiques avec des horizons plus engagés dans des parti-pris particuliers; mais, comme le souligne aussi R. Ogien, on ne voit pas bien ce que serait une théorie éthique sans application pratique, et je le suis entièrement sur ce point. Les anglophones n'ont pas de ces distinctions et, en philosophie analytique ou en philosophie morale dans les textes anglophones, les deux termes sont à peu près synonymes : moral philosophy ou ethics; morale ou éthique. J'ai donc choisi pour ma part, à la suite de R. Ogien mais aussi de M. Canto-Sperber par exemple (Canto-Sperber, 2001 [1996] ; Canto-Sperber \& Ogien, 2004), de les employer indifféremment.

Pourquoi existe-t-il une philosophie morale ? La question est évidemment immense mais on peut cependant faire une brève et très simple réponse : parce que, comme le social, le culturel, le psychologique, l'historique, le biologique, etc., la morale est une des composantes de la vie humaine et qu'on ne peut guère faire sans ${ }^{6}$. C'est le sens de la citation d'Emmanuel Lévinas que j'ai mise en exergue de cette présentation: la philosophie est morale. Quand on suppose une humanité du lien (ce que le rapport sur la morale laïque appelle faire communauté) et un vivre-ensemble acceptable, en même temps que l'existence de valeurs désirables, je ne vois pas comment on peut éradiquer la question de la vie bonne, de la vie heureuse et de la vie juste, du bien et du mal, de l'acceptable et de l'inacceptable, de la nuisance à soi-même et à autrui, et bien d'autres questions auxquelles ni la politique, ni la sociologie, ni la psychologie, ni l'histoire, ni l'économie, etc., ne peuvent (et, à mon avis, ne doivent) répondre. Et je ne vois pas non plus pourquoi l'école, qui est des principaux lieux de formation des individus, ne devrait pas toucher à cette notion-là, elle qui apprend à parler, à lire et à écrire aux petits d'humains, et qui devrait donc contribuer fortement à en faire des individus qui font communauté, qui vivent ensemble et qui considèrent que certaines façons d'être et de vivre sont désirables. Comme le dit Bouddha, qui accompagne Emmanuel Lévinas dans l'exergue, il existe un "parfum de l'homme éthique », ce qui implique que l'éthique est une dimension humaine, non forcément matérielle et palpable, mais diffuse et présente. 
Pour réfléchir à ces questions, on dispose de trois grands corps de pensée déjà anciens et de deux propositions plus récentes. Les trois grandes orientations sont bien connues : le déontologisme, dont le représentant principal est Kant, défendant une approche normative centrée sur des principes transcendants (les fameux "impératifs catégoriques ", non négociables); le conséquentialisme qui définit la vie bonne par la conséquence utile (l'utilitarisme de Mill en est la version la plus saillante, et R. Ogien qualifie d'ailleurs dans son ouvrage cette morale de laïque, pour montrer qu'en cette matière, la laïcité est plurielle) et l'éthique des vertus d'inspiration aristotélicienne, qui fait reposer la notion de bien sur la vertu des agents et la négociation intersubjective des valeurs, et non plus des normes. Récemment, et à partir du terreau féministe, a émergé l'éthique du care, courant souvent critiqué comme théoriquement faible car considéré comme simplement altruiste, voire naïf ou reposant sur des évidences, mais qui représente selon moi un des grands programmes moraux de notre contemporanéité (Gilligan, 2008 ; Tronto 2009 [1993]; Molinier et al., 2009). La question de la dimension morale du féminisme, non explicitement posée dans ce corpus éthique comme ailleurs, constitue d'ailleurs un impensé qui fait l'objet d'un article d'Anne-Charlotte Husson dans ce numéro. Enfin, il faut mentionner l'éthique minimale que R. Ogien élabore en 2003 à partir d'une réflexion sur la pornographie (Ogien, 2003), et qui postule un principe moral unique, celui de non-nuisance à autrui.

\section{Objectifs du numéro et présentation des articles}

20 L'objectif de ce numéro est d'interroger la dimension morale des/dans les formes langagières et discursives, des textes littéraires, des discours d'enseignementapprentissage, telle qu'elle s'y présente actuellement, en France en ce début de $\mathrm{XXI}^{\mathrm{e}}$ siècle.

21 Comme signalé plus haut, la théorie morale a beaucoup évolué ces 50 dernières années et les alternatives anciennes un peu rigides se sont diluées grâce à des propositions nouvelles, qui ont complexifié et enrichi le paysage : le retour en force de l'éthique des vertus aristotélicienne, l'émergence de la théorie du care, l'élaboration de l'éthique minimale, ont achevé semble-t-il la dilution des grands dualismes et la reconnaissance des liens entre affect et intellect, éthique et cognitif, entre autres. Les grandes catégories de pensée sont de plus en plus envisagées comme ouvertes, hétérogènes et non discrètes, et la catégorie morale sera envisagée dans ce numéro comme ouverte sur l'épistémique, l'affectif, le social, le politique, etc.

Du côté des circulations sociales, en France en tout cas, la morale en tant que telle et sous ce nom a été en revanche mise en sourdine par les impératifs de la démocratie et de la laïcité, et ce sont surtout, on vient de le voir, la notion et le nom éthique qui se manifestent dans les discours publics, à partir des questionnements bioéthiques et des problèmes environnementaux essentiellement. Mais le sens moral, quelle que soit l'origine et la forme qu'on lui accorde, demeure une faculté humaine, voire une disposition, et la question morale émerge régulièrement, à la faveur de débats ou d'événements discursifs : la réintroduction de la morale à l'école, la censure de certains textes, le retrait de certaines expositions, ou de certaines affiches, remettent régulièrement en jeu et au travail la question morale dans nos sociétés. 

langue et du discours, aux œuvres littéraires, et aux discours et postures pédagogiques. Ces trois domaines, présentés séparément dans la proposition que j'ai faite aux auteur-e$\mathrm{s}$, et qui devait dans mon esprit servir d'architecture au numéro, se sont entremêlés et reconfigurés dans les articles une fois écrits. Le sommaire se construit donc autrement, à partir de trois grands thèmes d'interrogation. l'enseignement. Le numéro est symboliquement ouvert par l'article d'une chercheuse, enseignante et formatrice belge, Claudine Leleux, qui jette d'emblée un éclairage exogène sur une question qui risque toujours de succomber à la fermeture d'un traitement franco-français. Dans «Instruire et éduquer sur fond d'éthique », elle montre quels sont les principes, les enjeux et les méthodes de l'enseignement de la morale à l'école en Belgique. L'article suivant, de Vincent Lorius, qui est à la fois personnel de direction et enseignant-chercheur, traite précisément de la question de la morale laïque dans l'enseignement en France. Intitulé «Éduquer scolairement dans un monde pluraliste: opportunité d'une approche normative du cadre réglementaire ", il montre comment la question de la morale, des normes et des valeurs peut se traiter dans l'Éducation nationale en France, et donne peut-être une forme de réponse à la critique de R. Ogien mentionnée plus haut. Enfin, Anne Leclaire-Halté, dans un travail sur les valeurs dans les albums de littérature de jeunesse, «Valeurs et rapport texte/image dans l'album de littérature de jeunesse: étude d'un exemple, Le génie du pousse-pousse», s'appuie sur le lien entre le texte et l'image pour proposer une réflexion et des pistes didactiques pour l'éducation aux valeurs.

La deuxième partie du numéro rassemble des articles qui ont en commun de traiter la question de la vérité. Dans «Les diseurs de vérité ou de l'éthique énonciative. Parrèsiastes, messagers, whistleblowers, lanceurs d'alerte ", j'examine une lignée de figures d'énonciateurs qui, de l'Antiquité à nos jours, de Démosthène à Snowden, ont adopté une pratique de la vérité radicale et dangereuse, pour des raisons d'éthique personnelle. Gaëlle Labarta aborde la question de la vérité et du mensonge au sein d'un questionnement plus large, en traitant de "l'inacceptabilité morale des discours littéraires ». Elle montre qu'en matière de littérature, les normes de tolérance morale peuvent être d'une part spécifiques (la fiction n'est pas évaluée de la même manière que les discours sociaux empiriques), et d'autre part contextuelles (comme toutes les évaluations, l'évaluation morale voit ses critères modifiés par les évolutions sociales, historiques culturelles). Enfin André Petitjean, choisissant de réfléchir sur le mensonge au théâtre dans « Du mensonge et de sa problématisation : illustration à partir de l'œuvre de Bernard-Marie Koltès", montre comment le dramaturge élabore un discours du mensonge qui en transgresse les normes pragmatiques habituelles.

La dernière partie du numéro aborde la question de la dimension morale des discours en termes de responsabilité, de référenciation et de nomination. Sophie Moirand propose de mettre au travail dans l'analyse des discours les trois notions de référenciation, généralisation et responsabilité énonciative. Dans "Trois notions à l'épreuve de la dimension morale du discours ", elle montre que la place pour une éthique langagière est au niveau de l'interprétation, et non de l'évaluation des discours, interprétation charpentée par ces trois notions. L'article suivant d'Anne-Charlotte Husson, «Genre et violence verbale: l'exemple de "l'affaire Orelsan"», propose une réflexion sur l'articulation encore inédite entre genre et morale, sous l'angle du féminisme. Analysant 
l'événement discursif qu'a constitué l'ensemble des réactions autour de la chanson du rappeur Orelsan en 2009, l'auteure analyse les implicites moraux des positions féministes, le plus souvent traduits en termes sociaux et politiques. L'article de Marc Arabyan clôt cet ensemble. Intitulé "De "classe ouvrière" à "classes moyennes", une réfection terminologique du champ social, en France ", il analyse un phénomène de modification lexicale qui lui semble aussi une perte sociale importante, montrant, s'il en était encore besoin, à quel point les mots font le monde autant qu'ils sont faits par lui.

Le dossier se clôt sur l'article de Roselyne Koren, à laquelle j'avais proposé de rédiger une synthèse de son travail pour ce numéro. Il faut rappeler que Roselyne Koren a été la première à poser explicitement la question de l'éthique du discours en analyse du discours et en rhétorique, et que bien des réflexions ultérieures, même en désaccord voire en opposition avec elles, lui doivent un premier appui de pensée. Dans une veine miépistémologique, mi-méthodologique, elle retrace ce qu'elle appelle des « Pérégrinations d'une analyste du discours en territoire éthique ", revenant sur le point d'accroche de tous ses travaux : la " prise de position ».

On verra que les articles se situent très différemment par rapport à la saisie de la morale par la philosophie. Si Claudine Leleux, Vincent Lorius, Gaëlle Labarta ou Anne-Charlotte Husson fréquentent ce corpus, et pensent à partir de lui dans des proportions variées, Anne Leclaire-Halté, André Petitjean, Sophie Moirand ou Roselyne Koren travaillent dans des cadres disciplinaires différents; et l'on verra que Marc Arabyan prend un parti clairement politique et social, et pense la question morale presque en dehors d'ellemême, pour ainsi dire. La question des outils de la pensée morale dans d'autres disciplines que la philosophie morale reste donc ouverte : doit-on utiliser les outils de la pensée morale pour penser des questions morales? J'espère que les articles du numéro répondront, entre autres, à cette question.

\section{BIBLIOGRAPHIE}

ANGENOT, M. (1982) : La Parole pamphlétaire. Typologie des discours modernes, Paris, Payot.

BUTLER, J. (2004 [1997]) : Le pouvoir des mots. Politique du performatif, trad. de l'américain par C. Nordmann, Paris, Éd. Amsterdam.

CANTO-SPERBER, M. (dir.) (2001 [1996]) : Dictionnaire d'éthique et de philosophie morale, Paris, Presses universitaires de France (Puf).

CANTO-SPERBER, M. \& OGIEN, R. (2004) : La philosophie morale, Paris, Puf.

GILLIGAN, C. (2008) : Une voix différente. Pour une éthique du care, trad. de l'américain par A. Kwiatek, Paris, Flammarion (coll. « Champs»).

LELIÈVRE, C. (2012) : «Vers quel enseignement d'une morale laïque? », Histoire et politique scolaire [blog], 3 sept. (en ligne : http://blogs.mediapart.fr/blog/claude-lelievre/030912/vers-quelenseignement-dune-morale-laique, consulté le 30/03/15). 
Ministère de l'Éducation nationale (2013) : Morale laïque. Pour un enseignement laïque de la morale. Remise du rapport de la mission sur l'enseignement de la morale lä́que, Lundi 22 avril 2013, Paris, ministère de l'Éducation nationale (en ligne : http://www.education.gouv.fr/cid71583/moralelaique-pour-un-enseignement-laique-de-la-morale.html, consulté le 30/03/15).

MOLINIER, P., LAUGIER, S. \& PAPERMAN, P. (2009) : Qu'est-ce que le care ? Souci des autres, sensibilité, responsabilité, Paris, Petite Bibliothèque Payot.

OGIEN, R. (2003) : Penser la pornographie, Paris, Puf.

- (2013) : La guerre aux pauvres commence à l'école, Paris, Grasset.

PAVEAU, M.-A. (2013) : Langage et morale. Une éthique des vertus discursives, Limoges, Lambert-Lucas. TRONTO, J. (2009 [1993]) : Un monde vulnérable, pour une politique du care, trad. de l'anglais par H. Maury, Paris, Éd. La Découverte.

\section{NOTES}

1. V. Peillon : « Je veux qu'on enseigne la morale laïque », Le JDD, 01/09/12.

2. "Enseignement de la morale: "Une nostalgie de l'école de la $\mathrm{III}^{\mathrm{e}}$ République" ", Le Monde, 03/09/12.

3. Le rapport est le fruit du travail d'Alain Bergounioux (inspecteur général de l'Éducation nationale, professeur associé à l'Institut d'études politiques de Paris) Laurence Loeffel (professeure en sciences de l'éducation à l'Université Charles de Gaulle-Lille 3) et Rémy Schwartz (ancien conseiller d'État et professeur associé à l'Université de Paris 1 Sorbonne).

4. Je reviens plus bas sur la question classique de l'emploi des termes éthique et morale.

5. Et je laisse de côté le troisième terme de déontologie, qui complique encore les circuits lexicaux, sémantiques et idéologiques de ces dénominations.

6. On m'objectera qu'il existe des individus amoraux mais c'est justement de manière privative qu'on les nomme, ce qui veut dire que leur comportement n'est pas considéré, dans la majeure partie des cultures et des sociétés, comme étant totalement acceptable.

\section{AUTEUR}

\section{MARIE-ANNE PAVEAU}

Pléiade (EA 7338), Université Paris 13 